\title{
Desempeño de modelos hidráulicos 1D y 2D para la simulación de inundaciones
}

\author{
Luis Timbe Castro ${ }^{1}$, Patrick Willems ${ }^{2}$ \\ ${ }^{1}$ Grupo de Ciencias de la Tierra y del Ambiente, Universidad de Cuenca, Av. 12 de abril S/N, \\ Cuenca, Ecuador \\ ${ }^{2}$ Hydraulics Laboratory, Faculty of Engineering, Katholieke Universiteit Leuven, Leuven, Belgium \\ Autor para correspondencia: luis.timbe@gmail.com
}

Fecha de recepción: 8 de octubre de 2010 - Fecha de aceptación: 6 de enero de 2011

\begin{abstract}
RESUMEN
En la actualidad, la disponibilidad de modelos digitales de elevación digital (MDEs) para representar la superficie de la tierra permite el acoplamiento de modelos hidráulicos con sistemas de información geográfica (SIG) para determinar la extensión de una inundación y los niveles del agua en la llanura de inundación. Muchos estudios de mapeo de inundaciones se han realizado usando modelos hidrodinámicos unidimensionales y bidimensionales (1D/2D). Algunos autores indican que los modelos hidráulicos 2D son la tecnología de punta para la modelación de inundaciones. Sin embargo, éstos modelos tienen la desventaja de que su aplicación está limitada por los altos requerimientos de datos, hardware y software. A su vez, los modelos 1D son ampliamente usados y dan resultados precisos en el cauce principal del río, pero son menos exactos para modelar el flujo de desbordamiento sobre las márgenes hacia las planicies adyacentes. El objetivo de ésta investigación es evaluar la precisión de la modelación de crecidas en las llanuras de inundación, comparando una aproximación cuasi-2D usando un modelo 1D con un modelo 2D completo. Los modelos fueron probados para el río Dender en Bélgica para tres eventos de inundación históricos.
\end{abstract}

Palabras clave: Hidráulica de ríos, inundaciones, planicies de inundación, MDE, modelos hidrodinámicos, SIG

\begin{abstract}
Nowadays, the availability of high resolution Digital Elevation Models (DEMs) to represent the earth surface allows coupling of hydraulic models with Geographic Information Systems (GIS) to obtain the flood extent and water levels in floodplains. Many studies on flood mapping have been conducted using one or two dimensional (1D/2D) hydrodynamic models. Some authors affirm that 2D hydraulic models are the state of the art for river flood modeling. They have, however, the disadvantage that their application is constrained by the high requirements for data, hardware and software. 1D models are capable of modeling precisely the flow in main rivers, but are less accurate for modeling over bank flow. The aim of the investigation is to test the accuracy for river floodplain modeling comparing a quasi-2D approach using a 1D model with a full 2D approach. The models were tested for the river Dender in Belgium for three historical flood events.
\end{abstract}

Keywords: River hydraulics, floods, floodplains, DEM, hydrodynamic models, GIS

\section{INTRODUCCIÓN}

Alrededor del mundo hay un creciente interés en la modelación de crecidas de ríos debido a los eventos de inundación extremos ocurridos en la última década (Badji y Dautrebande, 1997; Van der Sande y col., 2003; Nachtnebel, 2003). Al ser las inundaciones uno de los riesgos naturales más 
costosos, su análisis y prevención es un factor clave para las autoridades y organismos relacionados a los recursos hídricos. Los modelos de hidráulica de ríos usados para la modelación de inundaciones pueden clasificarse como modelos hidrodinámicos 1D o 2D. Los modelos hidrodinámicos 1D son ampliamente usados, estos modelos están basados en las ecuaciones de San Venant (leyes de conservación de la masa y momento) para calcular la superficie libre del agua para flujo estacionario y no estacionario en canales abiertos. Estas ecuaciones diferenciales parciales se resuelven mediante discretización numérica, usando frecuentemente el método de diferencias finitas, y en algunos casos mediante elementos finitos o volúmenes finitos, en un esquema implícito. Por otra parte, en los modelos hidrodinámicos 2D las ecuaciones de conservación de la masa y momento son expresadas en dos dimensiones y los resultados se calculan en cada punto de la malla en el dominio de solución. Los modelos 2D pueden resolverse usando el método de los elementos finitos. Muchos estudios de mapeo de inundaciones han sido realizados usando modelos hidrodinámicos 1D y 2D (Ahmad y Simonovic, 1999; Horritt y Bates, 2002; Bate y col., 1997; Sinnakaudan y col., 2002). Como afirma Mason y col., (2002), los modelos hidráulicos 2D son el estado del arte para la modelación de inundaciones. Aunque los modelos 1D son precisos en el cauce principal del río, éstos no son exactos para el flujo sobre los bancos (Bates y col., 1997), por ejemplo para la modelación de la onda de propagación desde el río hacia la llanura de inundación. Estos problemas no existen en los modelos 2D, sin embargo tienen la desventaja de requerir un mayor tiempo de implementación, y una alta demanda de datos y tiempo computacional.

Una solución intermedia entre la modelación 1D y 2D es una aproximación cuasi-2D. En este método, las llanuras de inundación son modeladas como ramales de río separados y conectadas al cauce principal por medio de vertederos o estructuras de desbordamiento. Esto permite al flujo de agua desbordar sobre la planicie de inundación cuando el nivel del agua excede los bancos o diques del río. Aunque muchos estudios usando modelos 1D/2D se han aplicado exitosamente para modelar crecidas, hay poca información sobre confiabilidad y precisión de los resultados del modelo. El objetivo de ésta investigación está dirigido a evaluar la precisión en la modelación de planicies de inundación, comparando una aproximación cuasi-2D (usando el modelo 1D) y un modelo completamente 2D. El modelo hidrodinámico fue calibrado y validado para tres eventos de crecida históricos en la cuenca del río Dender (Bélgica).

\section{MATERIALES Y MÉTODOS}

\subsection{Descripción del sitio y datos}

El área de estudio está ubicada en la parte alta de la cuenca del río Dender en la región Flamenca de Bélgica. Un tramo de aproximadamente $4800 \mathrm{~m}$ del río Dender que se inunda frecuentemente, aguas arriba de la estructura hidráulica de Geraardsbergen (región Overboelare), se usó para evaluar el desempeño de los modelos 1D (cuasi-2D) y 2D para la modelación de crecidas (Figura 1). Para la implementación del modelo se dispone de secciones transversales del río aproximadamente cada 50 m; mientras que para las llanuras de inundación existe un Modelo Digital de Elevación (MDE) basado en altimetría laser (LiDAR), con una resolución horizontal de $4 \mathrm{~m}$ (Figura 1). El MDE se corrigio en la parte superior de las márgenes usando el levantamiento de las secciones transversales. En el marco del proyecto FAME realizado para la Agencia Espacial Europea se realizó una nueva clasificación de uso del suelo en base a imágenes IKONOS tomadas en junio de 2002, dando como resultado un mapa de uso de suelo con una resolución de $4 \mathrm{~m}$ (Campling y col., 2002). En la región de Overboelare predomina bosque/arbustos y pastos/praderas, con un $35,2 \%$ y $27,5 \%$ respectivamente. También se dispone de mapas de inundación históricos desde 1988 a 2002. Estos mapas llamados "ROG" presentan las zonas que fueron inundadas en la región Flamenca y están basados en datos históricos (MVG, 2003; SADL, 2000). Se derivó una elevación media de inundación usando el límite del perímetro de los mapas ROG. Esta elevación media de inundación fue considerada en MIKE 11/MIKE 11 GIS, obteniendo así mapas ROG corregidos en formato raster. La corrección fue necesaria ya que normalmente los mapas ROG no siguen la isolineas topográficas, debido a que las diferentes fuentes de información usadas para su delineación (ej. reportes de campo, fotos aéreas y 
videos, etc.) no toman en cuenta de forma explícita la topografía. En los límites del modelo, se dispone de series temporales horarias de caudales y niveles observados, que son considerados en el modelo como condición de frontera.

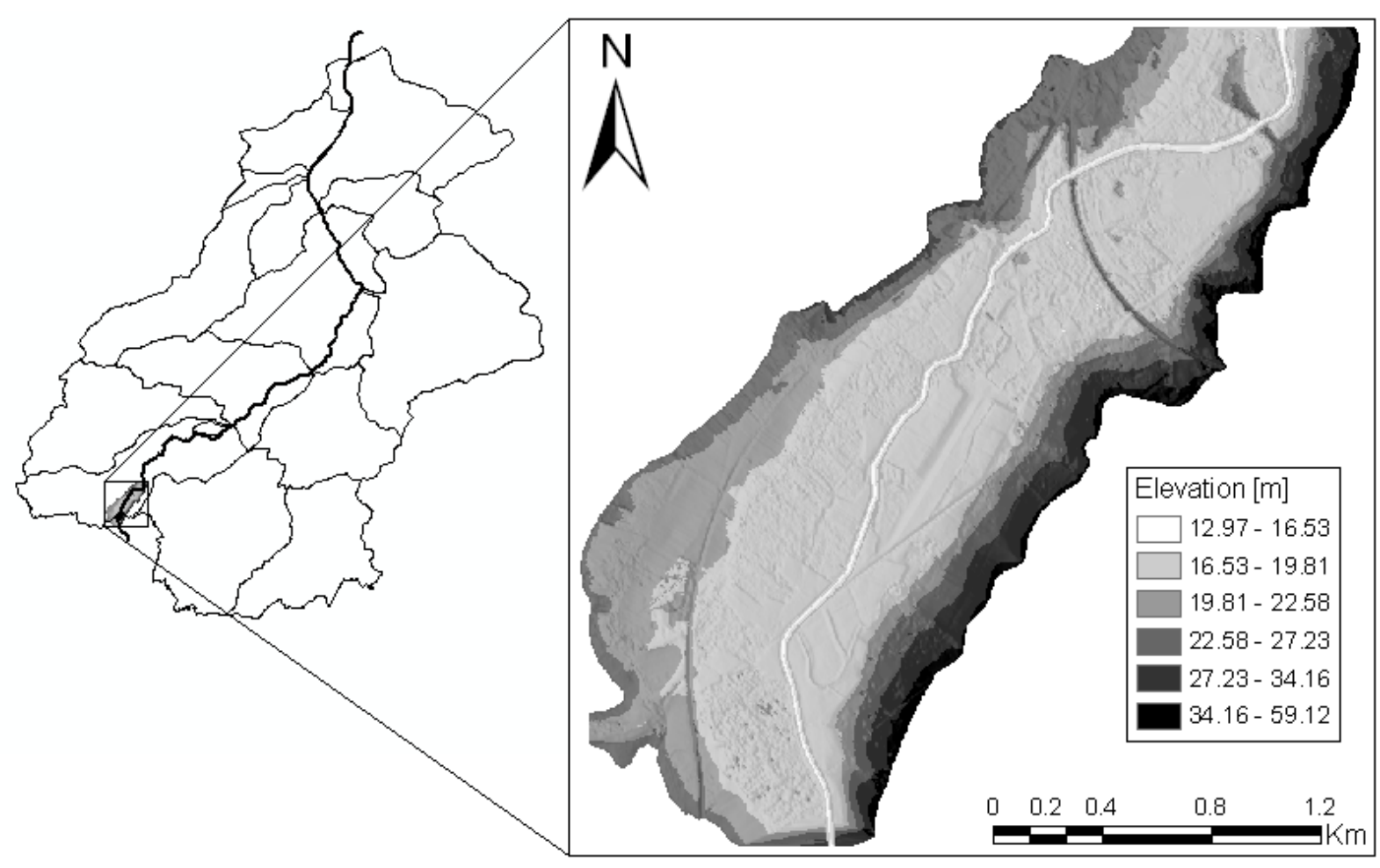

Figura 1. Ubicación de la zona de Overboelare en la cuenca del Dender y el MDE de las llanuras de inundación.

\subsection{Esquematización del modelo}

\section{MIKE 11/MIKE 11 GIS}

Estudios previos del río Dender han dado lugar a un modelo cuasi-2D para la modelación de crecidas, usando el paquete comercial MIKE 11 (Willems y col., 2001; Willems y col., 2002a; Willems y col., 2002b). MIKE 11 es un sistema 1D que resuelve las ecuaciones de San Venant usando un esquema de diferencias finitas implícito para el cómputo de flujo no estacionario en ríos y estuarios (DHI, 2002a).

Se usó un modelo cuasi-2D para enlazar el cauce principal con las planicies de inundación para la región de Overboelare. En esta aproximación, las planicies de inundación son modeladas como una red de ramales de río ficticios (en lo sucesivo llamados "ramales de inundación") y áreas de rebose (desbordamiento) entre el río principal y los ramales de inundación. Los ramales de río representan la depresiones topográficas (planicies de inundación), mientras que los reboses representan las elevaciones topográficas (diques, bancos, vías, líneas férreas). Un ramal de inundación se ha implementado en la margen izquierda, mientras que en la margen derecha fueron necesarios dos ramales de inundación para representar adecuadamente las características topográficas más complejas en esta zona. Las secciones transversales para los ramales de inundación fueron extraídas del MDE de alta resolución. El número de secciones transversales para los ramales de inundación fue seleccionado tratando de representar la topografía de las planicies lo más preciso posible. El mapeo de inundación se realizó usando la interface MIKE 11 GIS (DHI, 2002b). Las profundidades de inundación son calculadas en base a los niveles de agua a lo largo de los ramales de río, usando una rutina de extrapolación.

\section{MIKE FLOOD}


MIKE FLOOD integra los modelos 1D (MIKE 11) y 2D (MIKE 21) en un sistema único, acoplados dinámicamente (DHI, 2003a). El modulo hidrodinámico (HD) en el modelo de flujo de MIKE 21 está basado en las ecuaciones de San Venant en dos dimensiones, las cuales son resueltas mediante aproximaciones de diferencias finitas y un esquema de dirección implícita alternada en una malla regular o no estructurada (DHI, 2003b).

El modelo para la región de Overboelare usando MIKE FLOOD fue implementado usando la opción de enlace lateral, el cual permite a MIKE 11 una simulación 1D precisa en el cauce del río; y se usa MIKE 21 para la simulación 2D cuando el agua rebosa desde los bancos hacia la llanura de inundación. En la etapa inicial, el MDE original con una resolución de $4 \mathrm{~m}$ fue usado para implementar el modelo. Con esta resolución el modelo presenta problemas de estabilidad y fue posible conseguir una simulación exitosa únicamente con un intervalo de tiemplo de 1 segundo. Para superar este problema se tuvo que incrementar la resolución del MDE a $8 \mathrm{~m}$. Con ésta nueva resolución las elevaciones del MDE a lo largo de los bancos del río fueron promediadas, lográndose resultados mucho más estables. MIKE FLOOD usa éstas elevaciones para acoplar MIKE 11 y MIKE 21 a través de enlaces laterales. En consecuencia la variabilidad en las elevaciones de los bancos basados en el MDE afecta claramente la estabilidad del modelo y por lo tanto el intervalo de tiempo de simulación. Luego de cambiar la resolución del MDE, los pixeles a lo largo de los bancos fueron corregidos utilizando los puntos de los bancos de las secciones transversales medidas. De esta forma el modelo del cauce del río y los niveles de los bancos en el modelo 2D pueden considerarse comparables al modelo 1D con ramales de inundación y canales de enlace (aproximación cuasi-2D). Con éste procedimiento el intervalo de tiempo pudo incrementarse hasta 4 segundos.

\subsection{Evaluación de la extensión de inundación}

Para medir el ajuste entre los mapas de inundación históricos (observados) y simulados (modelados) se uso el índice de bondad-de-ajuste F presentado en la Ecaución (1) (Horrit y Bates, 2002; Bates y De Roo, 2000). El valor del índice F se encuentra en el rango [0-1] cumpliendo el criterio general para medir el desempeño del modelo. El numerador representa los pixeles comunes en ambos mapas: observado y simulado, mientras que el denominador es la unión de ambos mapas. F es igual a cero cuando no hay traslape entre el área inundada observada y simulada, y es igual a uno cuando ambas áreas inundadas son exactamente las mismas.

$$
F=\frac{A_{o b s} \cap A_{\mathrm{mod}}}{A_{o b s} \cup A_{\mathrm{mod}}}
$$

donde: $A_{o b s}$ y $A_{\text {mod }}$ representan el número total de pixeles inundados observados y simulados respectivamente.

\section{RESULTADOS Y DISCUSIÓN}

El mapeo de inundaciones en ambos modelos, cuasi-2D y 2D, fue realizado usando un MDE con una resolución de $8 \mathrm{~m}$ debido a limitaciones computacionales en MIKE FLOOD. Los modelos fueron evaluados y calibrados para los eventos de inundación históricos de diciembre 1993, enero 1995 y diciembre 2002. El coeficiente de rugosidad de Manning en MIKE 11 fue calibrado mediante el procedimiento de prueba y error, con valores tomados de literatura; obteniéndose valores calibrados de 0,03 para el lecho del río y 0,4 para las llanuras de inundación. Por otro lado, en MIKE FLOOD, el coeficiente de Manning obtenido fue de 0,04 para el lecho del río, y para las llanuras de inundación un valor promedio de 0,067 en base al mapa de uso de suelo.

No fue posible usar un mapa de rugosidad para simular el modelo 2D debido a problemas de estabilidad. El mapa de rugosidad fue derivado en base al mapa de uso de suelo. En algunos casos hay 
una gran variabilidad entre los valores de Manning en los límites entre dos clases de uso de suelo, lo cual podría causar los problemas de estabilidad. La Figura 2 presenta los resultados del mapa de inundación para el evento de diciembre 2002 para ambos modelos, y también muestra el mapa ROG. Una inspección visual de estos mapas demonstra en general una buena correspondencia entre los dos modelos, en términos de área inundada.

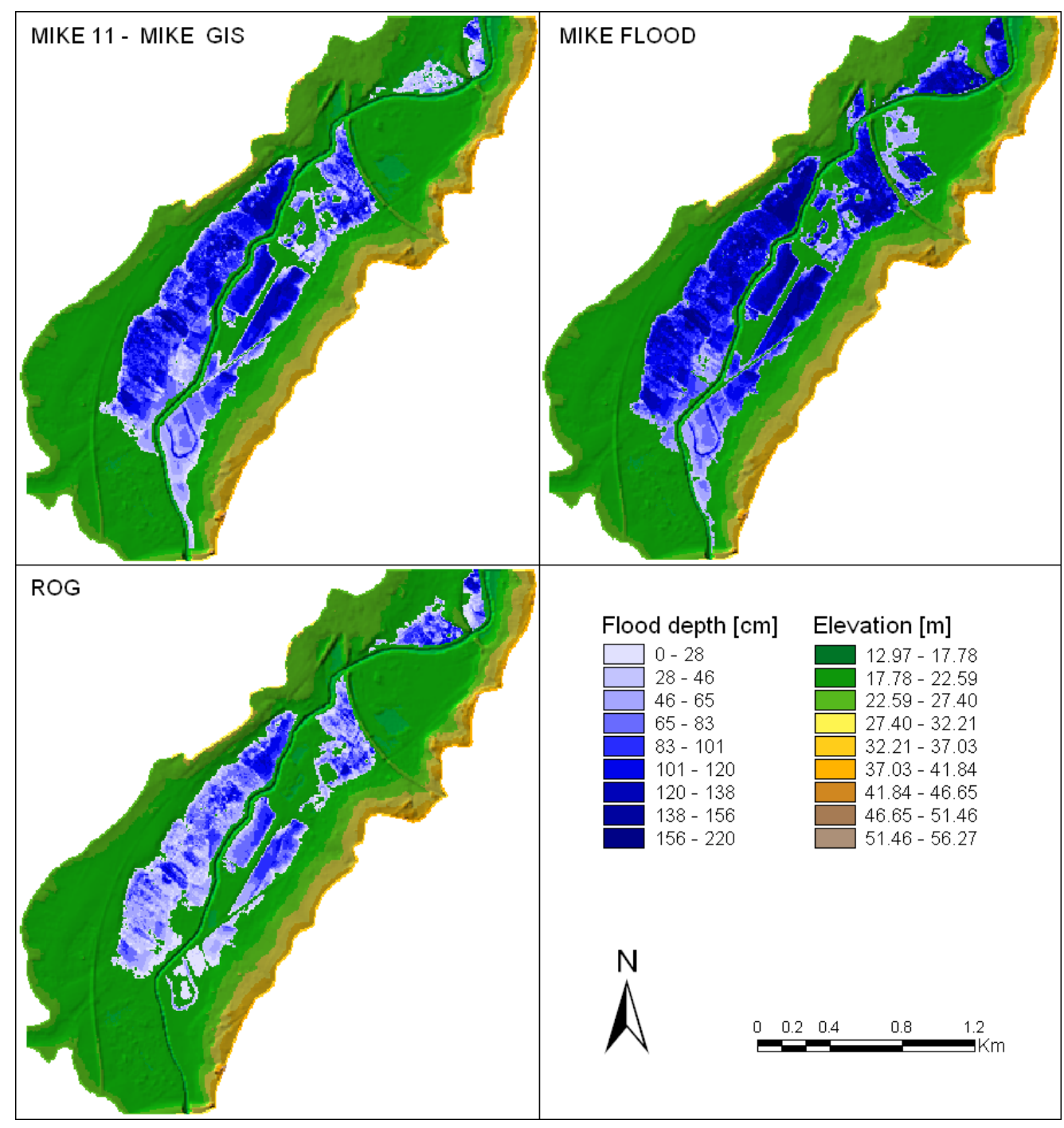

Figura 2. Mapas de inundación modelados y observado del evento de diciembre de 2002.

La Tabla 1 presenta los resultados del índice de bondad-de-ajuste $\mathrm{F}$, siendo éste parámetro el más apropiado para evaluar la coincidencia de las áreas inundadas. Este índice es igual a 1 (coincidencia del 100\%) solo si todos los pixeles inundados están ubicados en los mismos lugares en ambos mapas. Los dos modelos tienen un desempeño similar para aplicaciones de mapeo de inundaciones, con valores de $\mathrm{F}$ altos en ambas márgenes, variando entre $86,8 \%$ y $92,5 \%$ para las inundaciones históricas de enero 1995 y diciembre 2002. Para la crecida de diciembre 1993, la concordancia es más limitada, con un valor de $\mathrm{F}$ de $21,5 \%$ en la planicie derecha. Aunque en este último caso las áreas inundadas son similares, estas están ubicadas en lugares diferentes. Si comparamos los resultados del modelo MIKE 11/MIKE 11 GIS y los mapas ROG, hay un buen ajuste en la planicie izquierda, con un valor promedio de $\mathrm{F}$ de $88 \%$ para los dos eventos más extremos, mientras que para la llanura de inundación derecha el ajuste es ligeramente inferior, con un valor promedio de $\mathrm{F}$ de $71 \%$. En términos de área inundada, se concluye que para todas las inundaciones analizadas los dos modelos presentan valores similares. Si se comparan con los mapas ROG las diferencias son mayores. 
Tabla 1. Índice de bondad de ajuste F presentado como \% para los diferentes eventos de inundación en las márgenes izquierda y derecha.

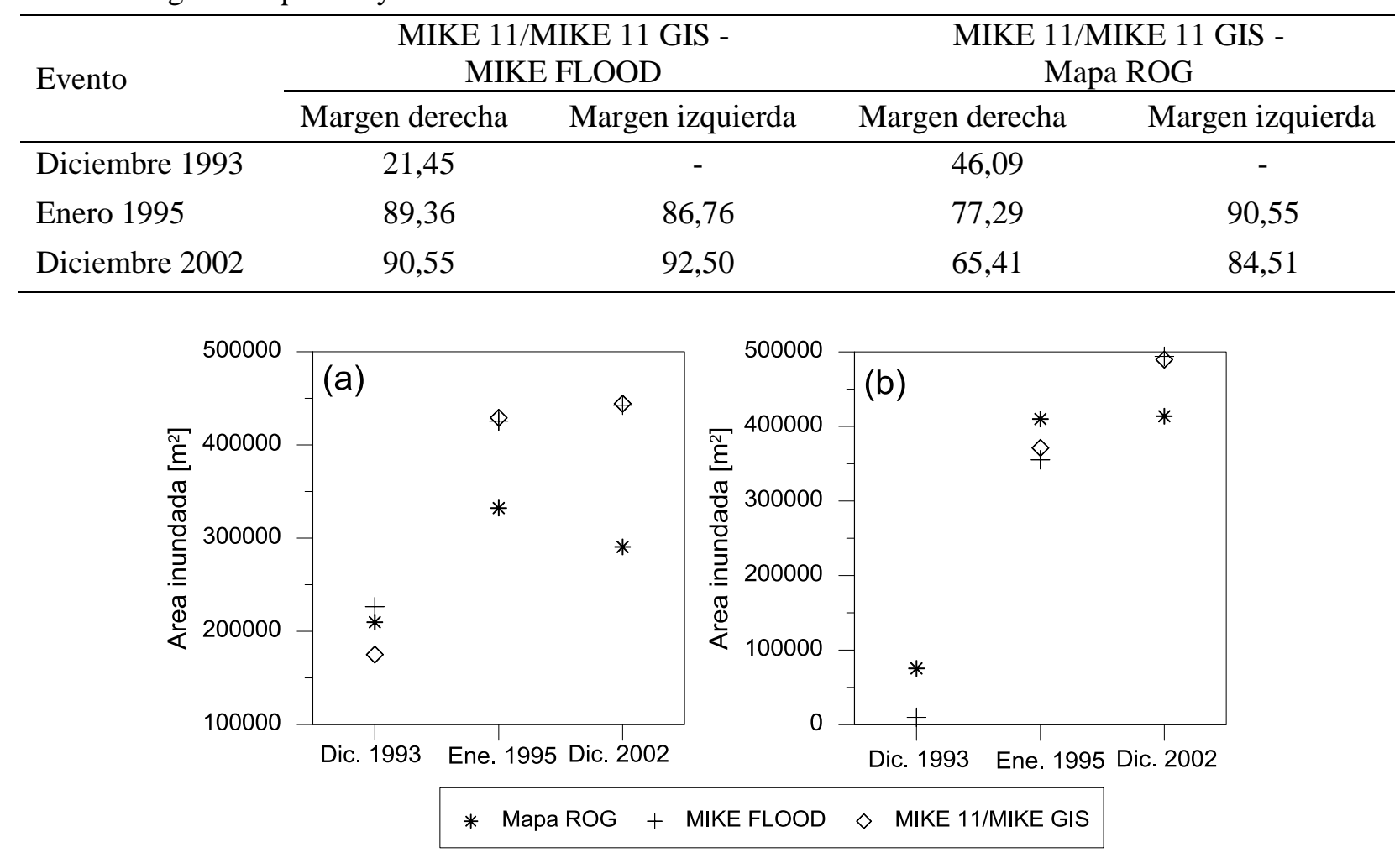

Figura 3. Áreas inundadas históricas simuladas y observadas en la margen (a) derecha, (b) izquierda.

La Figura 3 resume las áreas inundadas de los tres eventos de inundación analizados, en general ambos modelos presentan resultados similares en ambas márgenes. En la margen derecha las áreas inundadas usando MIKE 11/MIKE 11 GIS y MIKE FLOOD son casi iguales para los eventos de enero de 1995 y diciembre de 2002, mientras que las áreas inundadas según el mapa ROG son menores. El evento de diciembre de 1993 tiene menor magnitud que los otros dos, y las áreas inundadas son mucho menores, sin embargo estas varían dentro del mismo rango en los tres mapas. La margen izquierda muestra una menor variación de las áreas inundadas, de acuerdo al modelo MIKE 11/MIKE 11 GIS, esta planicie no se inunda en diciembre de 1993; también el modelo MIKE FLOOD presenta solo una pequeña área inundada cercana a cero, por lo tanto se puede considerar que esta zona no será inundada para este evento. En este caso, la pequeña área inundada presente en el mapa ROG podría estar sobrestimada dado que el cálculo de las profundidades de inundación fueron derivadas en base del perímetro de los mapas ROG originales o podría ser que la inundación fue producida por cauces tributarios presentes en ésta zona, los cuales no están implementados en el modelo. Los otros dos eventos de inundación en esta margen muestran una buena correspondencia de resultados entre los modelos y el mapa ROG.

\section{CONCLUSIONES}

Se han comparado las aproximaciones entre un modelo cuasi-2D (usando un modelo 1D) y un modelo completamente 2D para aplicaciones de modelación de inundaciones, a través de su implementación en los paquetes de modelación MIKE 11/MIKE 11 GIS y MIKE FLOOD. Se encontró que hay un buen ajuste en términos de área inundada entre ambos modelos y también con los mapas ROG 
corregidos. Los resultados muestran que el modelo MIKE FLOOD calcula profundidades de inundación mayores para alcanzar la misma área inundada en comparación a MIKE 11/MIKE 11 GIS; ésta discrepancia en las profundidades de inundación podría deberse a los diferentes métodos usados para la modelación de las planicies de inundación. MIKE 11 GIS hace un interpolación/extrapolación de los niveles de agua calculados en el ramal de inundación, con mayores profundidades aguas arriba que aguas abajo. Por el contrario, en MIKE 21 en el momento pico el nivel superficial del agua es casi horizontal, aún al inicio de la inundación se presenta esta condición. Un pixel se vuelve parte del área inundada una vez que ese pixel es hidráulicamente inundado. Esto explica los resultados de niveles de inundación más altos y relativamente constantes en MIKE 21. En general se puede concluir que grado de ajuste medido a través del índice $\mathrm{F}$ es mejor para los eventos de inundación de mayor magnitud (enero 1995 y diciembre 2002); mientras que para los eventos pequeños (diciembre 1993) en los cuales la llanura de inundación se llena parcialmente disminuye el porcentaje de ajuste.

La principal restricción en MIKE FLOOD son los altos requerimientos de hardware, resultando en un tiempo computacional considerable. Esta restricción se explica por la técnica de integración entre el modelo hidráulico y el SIG. MIKE11/MIKE 11 GIS es un ejemplo típico de una integración de acoplamiento libre, el modelo hidráulico y el SIG están acoplados usando una interface común para intercambiar los resultados del modelo en formato ASCII o binario. Esta configuración permite importar los resultados del modelo hidráulico en el SIG y el usuario puede crear los mapas de inundación en un tiempo específico o simultáneamente en varios momentos. Por el contrario, en MIKE FLOOD, el SIG está embebido dentro del modelo hidráulico 2D. Todas las capacidades necesarias de SIG en términos de manejo de datos y visualización están implementadas en el modelo hidráulico. El sistema se resuelve para el dominio completo del modelo en cada intervalo de tiempo; por lo tanto, se obtiene un mapa de inundación para cada paso de tiempo (ej. en este estudio cada 4 segundos), por lo tanto el tiempo computacional se incrementa exponencialmente. Este problema podría reducirse incrementando la resolución del DEM, pero esto igualmente puede ocasionar problemas de estabilidad y debe realizarse con cuidado, especialmente a lo largo de los bancos, donde los modelos MIKE 11 Y MIKE 21 son acoplados con la opción de enlace lateral. Los resultados sugieren que el modelo MIKE FLOOD puede aplicarse únicamente en áreas de interés específicas y para un número limitados de eventos de crecida específicos.

Para el caso especifico del Dender, en el cual los flujos bi-direccionales son más bien limitados, la precisión del modelo 2D en comparación con el modelo cuasi-2D no aumenta significativamente. Por lo tanto, el modelo cuasi-2D en este caso debería preferirse para áreas grandes y simulaciones continuas de largo plazo.

\section{AGRADECIMIENTOS}

Este estudio fue apoyado por el Selective Bilateral Agreements with Latin American Universities (K.U.Leuven), a través de la beca de doctorado de L. Timbe y por la Fund for Scientific ResearchFlanders (F.W.O.-Vlaanderen), a través de beca postdoctoral de P. Willems. Los datos y el modelo cuasi-2D fueron puestos a disposición con el apoyo de proyectos para el Flanders Hydraulics Water Administration (WL Borgerhout). Gracias también al DHI por proporcionar la licencia de MIKE FLOOD.

\section{BIBLIOGRAFÍA}

Ahmad, S., S.P. Simonovic, 1999. Comparison of one-dimensional and two-dimensional hydrodynamic modeling approaches for Red river basin. Natural Resources Institute,. University of Manitoba, Canada.

Badji, M., S. Dautrebande, 1997. Characterization of flood inundation areas and delineation of poor drainage soil using ERS-1 SAR imagery. Hydrol. Process., 11, 1441-1450. 
Bates, P.D., M.S. Horrit, S.N. Smith, D. Mason, 1997. Integrating remote sensing observations of flood hydrology and hydraulic modeling. Hydrol. Process., 11, 1777-1795.

Bates, P.D., A.P.J. De Roo, 2000. A simple raster-based model for flood inundation simulation. $J$. Hydrol., 236, 54-77.

Campling, P., S. Thompson, P. Willems, L. Timbe, M. Barbieri, 2002. Flood risk and damage assessment using modeling and earth observation techniques (FAME). Report Dender case. ESADUP 2 Small Services Project, coordinated by K.U.Leuven, Division SADL and Hydraulics Laboratory, Leuven, Belgium.

DHI, 2002a. MIKE11 A modelling system for rivers and channels - Reference manual. DHI Water \& Environment, Hørsholm, Denmark.

DHI, 2002b. MIKE 11-MIKE 11 GIS: Floodplain mapping and analysis - User guide. DHI Water \& Environment, Hørsholm, Denmark.

DHI, 2003a. MIKE FLOOD 1D-2D MODELLING - User manual. DHI Water \& Environment, Hørsholm, Denmark.

DHI, 2003b. MIKE 21: Coastal hydraulics and oceanography - Hydrodynamic module, Scientific Documentation. DHI Water \& Environment, Hørsholm, Denmark.

Horritt M.S., P.D. Bates, 2002. Evaluation of 1D and 2D numerical models for predicting river flood inundation. J. Hydrol., 268, 87-99.

Mason, D.C., D.M. Cobby, M.S. Horritt, P.D. Bates, 2002. Two-dimensional hydraulic flood modeling using floodplain topographic and vegetation features derived from airborne scanning laser altimetry. EGS XXVII General Assembly, Nice, France.

MVG, 2003. Risk zones for flooding (in Dutch). Note by the Ministry for the Flemish Community, Brussels, Belgium.

Nachtnebel, H.P., 2003. New strategies for flood risk management after the catastrophic flood in 2002 in Europe. Third DPRI-IIASA International Symposium on Integrated Disaster Risk Management: Coping with Regional Vulnerability, 3-5 July 2003, Kyoto, Japan.

SADL, 2000. Mapping of the natural and recent flooding areas in Flanders (in Dutch). Report for the Ministry of the Flemish Community, Division Water, by K.U.Leuven, Division SADL, Leuven, Belgium.

Sinnakaudan, S.A., A. Ab Ghani, C.C. Kiat, 2002. Flood inundation analysis using HEC and ArcView GIS 3.2a. 5th International Conference on Hydroscience and Engineering, Warsaw, Poland.

Willems P., G. Vaes, J. Berlamont, K. Cristiaens, D. Popa, L. Timbe, J. Feyen, 2001. Methodology for river flood modeling by the quasi two-dimensional approach. World Water and Environmental Resources Congress, Orlando, USA.

Willems, P., L. Timbe, D. Popa, 2002a. Mike11 model Dender, version April 2002 (in Dutch). Report including the results of the research project 'Implementation of the quasi $2 D$ approach for floodplain modeling for the MIKE11 model of the river Dender' for the Flanders Hydraulics water administration (WL Borgerhout). Report by K.U.Leuven, Hydraulics Laboratory, Leuven, Belgium.

Willems, P., G. Vaes, D. Popa, L. Timbe, J. Berlamont, 2002b. Quasi 2D river flood modeling. En: Bousmar \& Zech (Eds.). River Flow 2002: International congress on Fluvial Hydraulics, Vol. 2. Louvain-la-Neuve, 4-6 September 2002, 1253-1259.

Van der Sande, C.J., S.M. de Jong, A.P.J. de Roo, 2003. A segmentation and classification of IKONOS-2 imagery for land cover mapping to assist flood risk and flood damage assessment. Int. J. Appl. Earth Obs. Geoinf., 4, 217-229 